\title{
Time-resolved electron-temperature measurement in a highly excited gold target using femtosecond thermionic emission
}

\author{
X. Y. Wang, ${ }^{*}$ D. M. Riffe, ${ }^{\dagger}$ Y.-S. Lee, and M. C. Downer \\ Department of Physics, The University of Texas, Austin, Texas 78712
}

(Received 3 June 1994)

\begin{abstract}
We report direct measurement of hot-electron temperatures and relaxation dynamics for peak electron temperatures between 3400 and $11000 \mathrm{~K}$ utilizing two-pulse-correlation femtosecond (fs) thermionic emission. The fast relaxation times $(<1.5 \mathrm{ps})$ are described by extending RT characterizations of the thermal conductivity, electron-phonon coupling, and electronic specific heat to these high electron temperatures.
\end{abstract}

Hot-electron relaxation dynamics have attracted intense interest ${ }^{1-3}$ since the invention of ultrafast laser sources. For low excitation levels (peak electron temperatures $k_{B} T_{\text {peak }}<0.2 \mathrm{eV}$ ) the main interest has been the extraction of the electron-phonon-coupling constant $G$. $^{1,2}$ Indeed this has been done for a variety of materials. In the higher excitation regime, above $k_{B} T_{\text {peak }}=0.2 \mathrm{eV}$, a variety of interesting phenomena occur which depend upon understanding either electron-relaxation dynamics or even simply the peak electron temperature under given experimental conditions. For example, in high-field nanoscale microelectronic devices, ${ }^{4}$ electron energies in excess of $1 \mathrm{eV}$ commonly arise. Simulations ${ }^{5}$ of these devices show that relaxation rates are directly affected by density-of-states (DOS) considerations; however, direct experimental evidence of such DOS effects is lacking. In fs-laser plasma physics the electron temperature $T_{e}(t)$ is critical to understanding the $x$-ray yield and pulse duration $^{6}$ as well as the thermodynamic conditions and transport properties of the laser-produced plasmas. $^{7}$ Reflectivity measurements aimed at deducing $T_{e}(t)$ have been performed. ${ }^{8}$ However, the extraction of $T_{e}(t)$ is not direct and must be inferred from phonon-coupling and diffusivity parameters which are unknown at high temperatures. That is, an order-of-magnitude difference in $T_{\text {peak }}$ has arisen in separate analyses ${ }^{9}$ of the data from the reflectivity experiment by Milchberg et al. ${ }^{8}$ Finally, in the seemingly unrelated area of surface physics, electrons with temperatures on the order of $1 \mathrm{eV}$ are key participants in the ultrafast photodesorption of adsorbates. ${ }^{10}$

In spite of this widespread interest in very hot electrons, remarkably absent are direct experimental investigations of electron dynamics and measurements of the electron temperature above $k_{B} T_{\text {peak }}=0.2 \mathrm{eV}$. Some of the reasons for this have been experimental, since the high-sensitivity thermomodulation ${ }^{1}$ or surface plasmon ${ }^{2}$ experiments widely used at low excitation levels are not easily transferable to higher power lasers with low repetition rates and nonlinear saturation effects. A potentially attractive method, due to its ease of implementation, has been laser-induced electron emission. ${ }^{3}$ The drawback to electron emission has been the perception that the strong space-charge fields associated with the large number of particles ejected in this temperature range preclude using it in a quantitative manner. However, recent work $^{11}$ for

$T_{e}(t)$ pulses $\lesssim 10 \mathrm{ps}$ in duration has shown that the yield from thermionic emission is a simple analytic function whose $T_{e}(t)$ dependence arises solely from the peak electron temperature $T_{\text {peak }}$ generated by the laser; thus there now exists the possibility of directly probing highexcitation-level electron dynamics.

In this Brief Report we illustrate, using data from an Au target, the direct extraction of electron cooling dynamics using a two-pulse correlation measurement of the total electron yield for excitations from $T_{\text {peak }} \approx 0.3 \mathrm{eV}$ up to the damage threshold at $T_{\text {peak }} \approx 1 \mathrm{eV}$. The measured rates are much faster than those expected using roomtemperature (RT) parametrizations of the thermal conductivity $\kappa$, electronic specific heat $C_{e}$, and $G$. By including DOS considerations (arising from the Au $5 d$ states in the present case), which produce a strong $T_{e}$ dependence on $G$ and $C_{e}$, and explicit dependence of $\kappa$ upon $T_{e}$ and the lattice temperature $T_{i}$, we have successfully modeled the measured cooling curves. Given the simplicity of the fs thermionic-emission technique, it should be widely applicable to investigating electron dynamics in a variety of materials over a wide temperature range.

A polycrystalline gold sample of 5000 - $\AA$ thickness was prepared by evaporation onto a clean $\mathrm{Si}$ wafer in a vacuum chamber of $\sim 10^{-6}$ Torr. It was then immediately placed in an ultrahigh-vacuum chamber which was maintained at a base pressure of $\lesssim 2 \times 10^{-10}$ Torr. The experiments were performed with 120-fs, 630-nm laser pulses generated by a colliding pulse mode-locked (CPM) dye laser and a $10-\mathrm{Hz}$, four-stage yttrium aluminum garnet (YAG) pumped dye amplifier. ${ }^{12}$ The amplified pulses are split into two nearly equal intensity beams and then recombined to form a collinear $p$-polarized beam incident at $68^{\circ}$ with a variable time delay $\Delta t$ between the two pulses. The spot size on the sample is $320 \times 220 \mu \mathrm{m}^{2}$. The absorbed energy density $u$ is determined from the absolute value of reflectivity $R\left(68^{\circ}\right)=0.91$, which equals that determined from published optical constants for Au. ${ }^{13}$ Below the damage threshold the dependence of $R$ on the laser intensity is negligible. Other details of the experimental conditions have been described previously. ${ }^{11}$

In the femtosecond laser intensity regime utilized here, the electron emission is dominated by the thermionic process which is strongly space charge suppressed. For 
emission times $\lesssim 10$ ps and peak electron temperatures $T_{\text {peak }}>0.3 \mathrm{eV}$, the total yield is essentially linear with respect to $T_{\text {peak }}$ and is given by ${ }^{11}$

$$
\begin{aligned}
N_{\text {esc }}=\frac{k_{B} T_{\text {peak }}}{a e^{2} / R_{1}} \log _{e}[1+ & C \tau \pi R_{2} a e^{2} k_{B} T_{\text {peak }} \\
& \left.\times \exp \left(-\frac{\varepsilon_{F}-\mu+e \Phi}{k_{B} T_{\text {peak }}}\right)\right],
\end{aligned}
$$

where $N_{\text {esc }}$ is the number of electrons that overcome the space-charge barrier and ultimately escape from the sample, $R_{1}$ and $R_{2}$ are the full width at half maximum FWHM radii of the elliptical spot size, $a$ is a geometric parameter close to $2, C=4 \pi m / h^{3}, \tau$ is the pulse duration for a full width at $80 \%$ of $T_{\text {peak }}$, and $\varepsilon_{F}$ and $\mu$ are the Fermi energy and chemical potential, respectively.

That the total yield depends solely on the peak value $T_{\text {peak }}$ of the electron temperature $T_{e}(t)$, but not the temporal details of the electron heating, has been demonstrated quantitatively by particle simulations, ${ }^{11,14}$ and can be understood qualitatively as follows. First, because thermionic emission is exponentially activated, most emitted electrons just above the metal surface are generated for temperatures very close to $T_{\text {peak }}$. Moreover, for thermionic emission on a time scale of $\lesssim 10 \mathrm{ps}$, the fastest electrons in the emitted distribution (which uniquely reflect $T_{\text {peak }}$ ) arrange themselves at the front of the electron pulse and thus experience the least spacecharge yield suppression while rejecting slower electrons back to the surface. Conversely, for emission time scales $\gtrsim 10 \mathrm{ps}$, the fastest electrons separate away from surface while cooler electrons are still being ejected. Hence space-charge suppression of slower electrons becomes less effective, and the temporal profile of $T_{e}$ becomes important in determining the total yield. Experimentally, as shown below, we find that when two heating pulses are

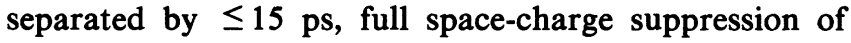
slower electrons by the faster electrons is maintained and Eq. (1) is thus valid.

From Eq. (1) and the preceding paragraph, it follows that the measured yield vs $\Delta t$ of two identical laser pulses approximately mirrors the electron temperature $T_{e}(t)$ generated by a single laser pulse of the same intensity. This is because $T_{\text {peak }}$, which is generated just after the second laser pulse, depends monotonically on the electron temperature $T_{e}$ at the time $\Delta t$ the second pulse arrives. For two overlapping pulses $T_{e}(\Delta t)$ is obviously a maximum, and therefore so is $T_{\text {peak }}$. As $\Delta t$ increases, $T_{e}(\Delta t)$ decreases, and therefore so does $T_{\text {peak }}$. For $\Delta t$ such that $T_{e}(\Delta t)$ has returned to near the ambient temperature, $T_{\text {peak }}$ (and hence the yield) for the two pulses is at a minimum (and is the same as for just one pulse). Hence by simply measuring the yield vs pulse separation one obtains an immediate approximate assessment of the hot-electron cooling curve for single-pulse excitation. ${ }^{15}$

Figure 1 shows the measured electron yield versus $\Delta t$ for various laser intensities. The yield drops to a constant value (from its maximum at zero time delay) in less than $500 \mathrm{fs}$ for excitation at a total zero-time-delay intensity of $I_{0}=1.0 \times 10^{11} \mathrm{~W} / \mathrm{cm}^{2}$ [Fig. 1(d)]. As $I_{0}$ increases, the cooling time increases, up to $\sim 1.5$ ps at $I_{0}=1.5 \times 10^{12} \mathrm{~W} / \mathrm{cm}^{2}$. The intensity ratio between the trailing and leading pulses is about 0.9. The data show only a small $(\sim 5 \%)$ discrepancy between the yield $N_{F}$ from two-pulse excitation at long delays ( $>1.5 \mathrm{ps})$ and the yield $N_{1}$ generated by the stronger pulse. This small discrepancy is caused by a slight mismatch between shapes of the two laser spots on the sample, creating a slightly larger excitation area for both pulses than when either single pulse is incident. Apart from this small error, however, $N_{F} \approx N_{1}$, as predicted. The inset in Fig. 1(c) shows a typical longer time-delay measurement, showing the yield recovering from the flat minima in the range $1.5 \leq \Delta t \lesssim 15$ ps (at $\Delta t>250$ ps the total yield reaches the yield sum from the two individual pulses), as discussed above.

Using Eq. (1) the electron yield data of Fig. 1 is converted into $T_{\text {peak }}$ and plotted vs $\Delta t$ in Fig. 2. At the highest intensities $T_{\text {peak }} \sim 0.95 \mathrm{eV}$ is achieved. The electron temperatures shown here are consistent with those determined from electron-energy distributions (not shown) which have also included space-charge effects in their analysis. ${ }^{11,14}$

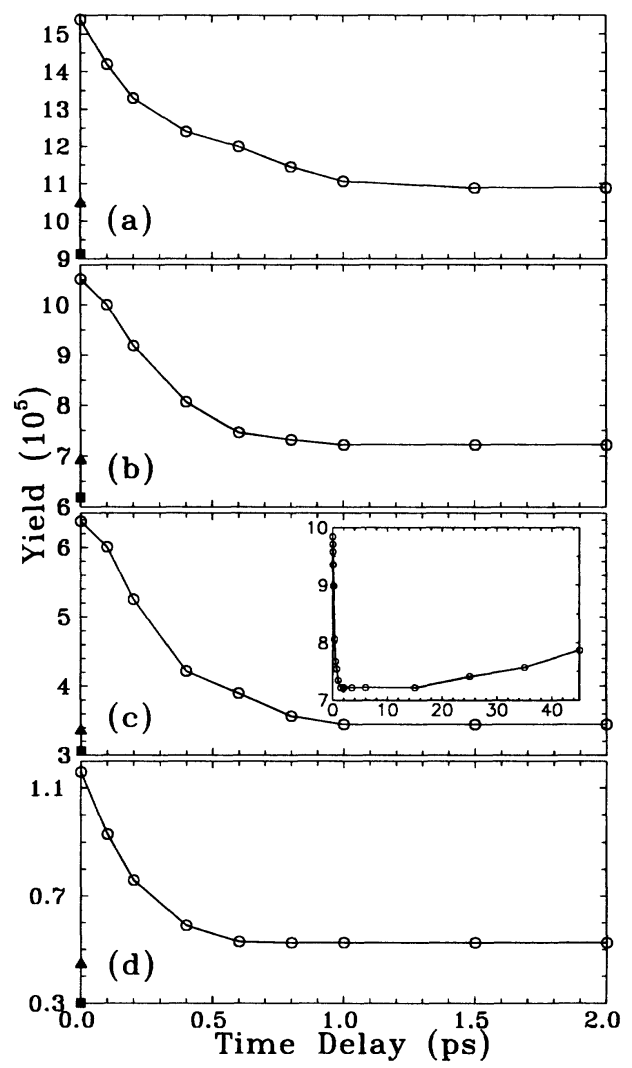

FIG. 1. Total electron yield (open circles; in units of $10^{5}$ electrons per pulse pair) vs time delay between the two laser pulses at various intensities. The yield produced by each individual pulse is given by the triangles and squares for the leading and trailing pulse, respectively. The ratio of the trailing and leading pulse intensities is $\sim 0.9$. The total intensities at zero time delay are (a) $1.5 \times 10^{12} \mathrm{~W} / \mathrm{cm}^{2}$, (b) $8 \times 10^{11} \mathrm{~W} / \mathrm{cm}^{2}$, (c) $4 \times 10^{11} \mathrm{~W} / \mathrm{cm}^{2}$, and (d) $1 \times 10^{11} \mathrm{~W} / \mathrm{cm}^{2}$. Inset: a typical longer time-delay measurement to illustrate the dissipation of space-charge effects for $\Delta t>15$ ps. 


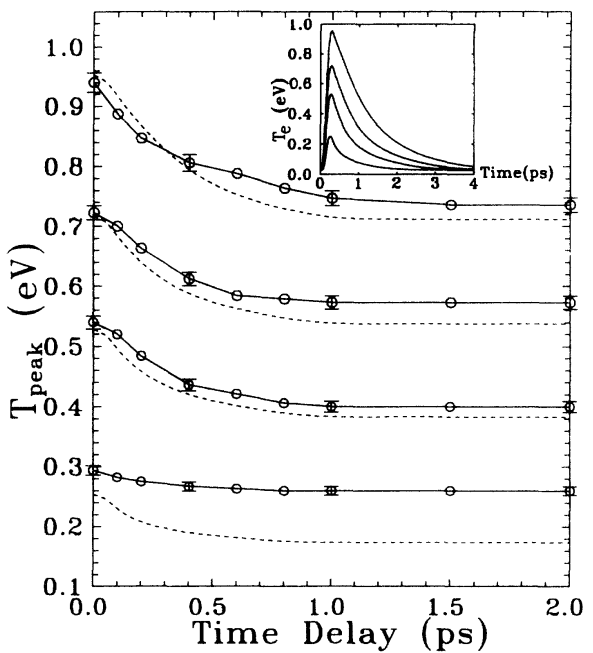

FIG. 2. Main panel: Peak electron temperature vs the time delay at overall intensities $1.5,0.8,0.4$, and $0.1 \times 10^{12} \mathrm{~W} / \mathrm{cm}^{2}$, respectively. The open circles and solid lines represent the data, and the dotted lines are the theoretical simulations with temperature dependent $C_{e}, G$, and $\kappa$. Inset: the simulated electron temperature evolution at corresponding zero-time-delay intensities.

In order to model the cooling dynamics from these two-pulse data, we use the following coupled nonlinear differential equations for $T_{e}$ and $T_{i}$ which account for the electron-phonon coupling and thermal conductivity of the sample:

$C_{e}\left(\partial T_{e} / \partial t\right)=\nabla\left(\kappa \nabla T_{e}\right)-G\left(T_{e}-T_{i}\right)+\partial u(r, t) / \partial t$,

$C_{i}\left(\partial T_{i} / \partial t\right)=G\left(T_{e}-T_{i}\right)$,

where $C_{e}$ and $C_{i}$ are the electron and lattice specific heats, respectively, and $u(r, t)$ is the absorbed energy density from the laser pulses. The equations assume that the electrons thermalize on a time scale shorter than the laser pulse, an approximation which is valid in this temperature regime. ${ }^{16}$ By using RT values of $C_{e}, \kappa$, and $G$ for $\mathrm{Au}$ $\left(C_{e}=\gamma T_{e}, \quad \gamma=67.6 \mathrm{~J} / \mathrm{m}^{3} \mathrm{~K}^{2}, \quad \kappa=315 \mathrm{~W} / \mathrm{mK},{ }^{17}\right.$ and $G=3.5 \times 10^{16} \mathrm{~W} / \mathrm{m}^{3} \mathrm{~K}^{1,2}$ ), equations (2) and (3) were solved for two laser pulses equivalent to those used in the experiment. [The temperature dependence of $C_{i}$ $\left(=2.5 \times 10^{6} \mathrm{~J} / \mathrm{m}^{3} \mathrm{~K}\right.$ (Ref. 17) at RT) is very weak below the damage threshold, has little effect on the overall cooling dynamics, and hence is neglected in the simulation.] The results, plotted in Fig. 3, show that $T_{e}(t)$ (produced for zero time delay, main panel) and hence $T_{\text {peak }}$ vs $\Delta t$ (inset) both decay on a time scale much longer than experimentally observed, and they also yield $T_{\text {peak }}$ 's considerably higher than the measurement indicates at high excitations. It is clear that these low-temperature values for $C_{e}, \kappa$, and $G$ are poor approximations for $\mathrm{Au}$ in this high-temperature regime.

The most severe problem has to do with the total neglect of the $5 d$ electron band [located between 7.4 and $2.2 \mathrm{eV}$ below $\varepsilon_{F}$ (Ref. 18)] in describing the high- $T_{e}$ properties of $\mathrm{Au}$. By accounting for the thermal excitation of the $5 d$ electrons, all three parameters $\left(C_{e}, \kappa\right.$, and $\left.G\right)$ are substantially affected. First, we calculate the temperature

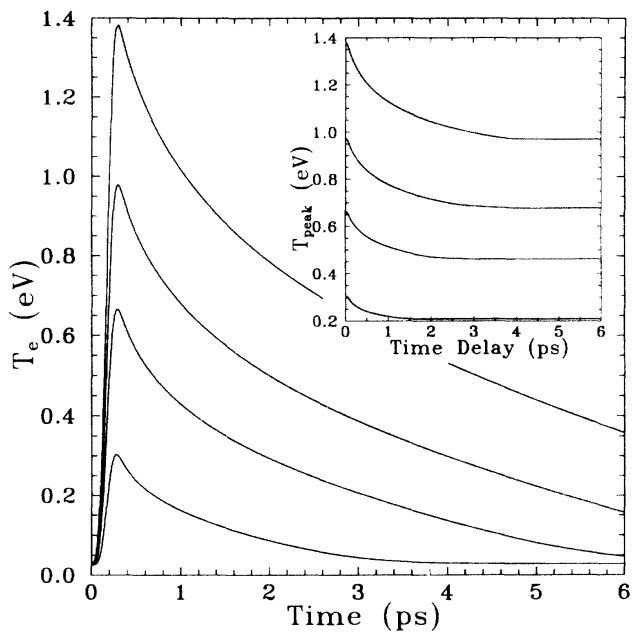

FIG. 3. Simulations using RT constants $G$ and $\kappa$ with $C_{e}=\gamma T_{e}$ for the corresponding experimental conditions given in Fig. 1. Main panel: the electron temperature evolution at zero-time-delay intensities; inset: the peak electron temperature vs the time delay between two pulses.

dependence of $C_{e}$ as in Ref. 11 using the band structure from Ref. 18. This results in a value of $C_{e}$ about 2.5 times larger than the RT relation $C_{e}=\gamma T_{e}$ would produce at $T_{e}=1 \mathrm{eV}$.

The temperature dependence of $G$ is calculated using the formalism of Allen, ${ }^{19}$ which describes the rate of energy exchange between electrons and phonons with two distinct temperatures as

$$
\begin{aligned}
C_{e}\left(\partial T_{e} / \partial t\right)_{e-\mathrm{ph}}=(4 \pi / \hbar V) \sum_{k k^{\prime}} & h \omega_{Q}\left|\boldsymbol{M}_{k k^{\prime}}\right|^{2} S\left(k, k^{\prime}\right) \\
& \times \delta\left(\varepsilon_{k}-\varepsilon_{k^{\prime}}+h \omega_{Q}\right) .
\end{aligned}
$$

Here $\boldsymbol{M}_{k k^{\prime}}$ is the electron-phonon-scattering matrix element, $V$ the sample volume, $\omega_{Q}$ the frequency of a phonon with momentum $Q$, and $\varepsilon_{k}$ the energy of an electron with momentum $k . \quad S\left(k, k^{\prime}\right)=f_{k}\left(1-f_{k^{\prime}}\right) n_{Q}-f_{k^{\prime}}(1$ $\left.-f_{k}\right)\left(n_{Q}+1\right)$ is the thermal statistical factor describing phonon emission and absorption via electron scattering where $f_{k, k^{\prime}}$ and $n_{Q}$ are Fermi and Bose-Einstein distribution functions for the electrons and phonons, respectively. A high- $T_{e}$ extension of Allen's equations requires one to account for the electron-energy dependence of the electron-phonon spectral density function $\alpha^{2} F\left(\varepsilon, \varepsilon^{\prime}, \Omega\right)$. Under most circumstances, when $T_{e} \sim \mathrm{RT}$, one is justified in substituting the Fermi energy $\varepsilon_{F}$ for $\varepsilon$ and $\varepsilon^{\prime}$ since only states near the Fermi energy are involved in electronphonon scattering. In order to explicitly account for the $5 d$ states we make the approximation that $\alpha^{2} F\left(\varepsilon, \varepsilon^{\prime}, \Omega\right)=\left[g(\varepsilon) g\left(\varepsilon^{\prime}\right) / g^{2}\left(\varepsilon_{F}\right)\right] \alpha^{2} F\left(\varepsilon_{F}, \varepsilon_{F}, \Omega\right)$, where $g(\varepsilon)$ is the electron density of states at energy $\varepsilon$. This is equivalent to assuming that the magnitude of $\boldsymbol{M}_{k, k^{\prime}}$ is independent of the electron states $k$ and $k^{\prime}$. Following Allen, but using our approximation for $\alpha^{2} F$, we find $G=\pi \hbar k_{B} \lambda\left\langle\omega^{2}\right\rangle g_{\varepsilon} / g\left(\varepsilon_{F}\right)$, where $g_{\varepsilon}=\int g^{2}(\varepsilon)$ $(-\partial f / \partial \varepsilon) d \varepsilon$ is temperature dependent at high temperatures. Near RT, $-\partial f / \partial \varepsilon \approx \delta\left(\varepsilon-\varepsilon_{F}\right)$, so that $g_{\varepsilon}(\mathbf{R T})$ reduces to $g^{2}\left(\varepsilon_{F}\right)$ and we recover the expression given by 
Allen: $G_{0}=\pi \hbar k_{B} g\left(\varepsilon_{F}\right) \lambda\left\langle\omega^{2}\right\rangle$. However, at high temperatures $-\partial f / \partial \varepsilon$ becomes non-negligible far away from $\varepsilon_{F}$. For excitation levels used in the experiment, $d$-band electrons are excited into the conduction band, resulting in $g_{\varepsilon}$ quite different from $g^{2}\left(\varepsilon_{F}\right)$. By including the $d$ band electrons, we find that $G$ at $k_{B} T_{e}=1 \mathrm{eV}$ increases by a factor of $\sim 6$ over $G$ at RT.

The thermal conductivity $\kappa$ depends on both the electron and lattice temperatures. In general one can write ${ }^{20}$ $\kappa=\left(\frac{1}{3}\right) v_{F}^{2} \tau C_{e}$, where $v_{F}$ is the electron Fermi velocity ${ }^{21}$ and $\tau$ is the electron relaxation time. For $T_{e}>\Theta_{D}$ (the Debye temperature) $\tau$ is the same $\mathrm{s}^{22}$ as the one in the electrical conductivity. Both electron-phonon and electronelectron scatterings contribute to the electron-relaxation time $\tau$ with $1 / \tau=1 / \tau_{e-\mathrm{ph}}+1 / \tau_{e-e}$, where $1 / \tau_{e-\mathrm{ph}}=B T_{i}$ and $1 / \tau_{e-e}=A T_{e}^{2}$. For Au, $A=1.2 \times 10^{7} \mathrm{~K}^{-2} \mathrm{~s}^{-1}, 23$ and $B=1.23 \times 10^{11} \mathrm{~K}^{-1} \mathrm{~s}^{-1} .{ }^{17}$ Ballistic transport ${ }^{24}$ of electrons away from the excited region is neglected here since it should be severely suppressed by the high scattering rate at high temperatures. Therefore, we write the thermal conductivity as $\kappa=\left(\frac{1}{3}\right) v_{F}^{2} C_{e}\left(T_{e}\right) /\left(B T_{i}+A T_{e}^{2}\right)$.

When we implant the above formalism for the temperature dependence of $C_{e}, \kappa$, and $G$ into Eqs. (2) and (3), and numerically solve for the peak temperatures with an input of two laser pulses at various time delays between them, we obtain the simulation results shown in Fig. 2 as dotted lines. Note that both the simulated absolute $T_{\text {peak }}$ 's (except at the lowest intensity) and cooling times agree very well with the experiment data. The inset shows the simulated $T_{e}(t)$ at the corresponding zerotime-delay intensities. The calculations show that the largest contribution to cooling arises from the temperature dependence of $\kappa$. The discrepancy with the data at the lowest excitation is caused by multiphoton and thermally assisted photoemission processes, ${ }^{3}$ which play considerable roles in the electron total yield at low intensities. ${ }^{11}$

In summary, we have performed direct time-resolved measurements of the electron cooling dynamics at electron temperatures up to about $1 \mathrm{eV}$, revealing the fast electron-energy relaxation $\lesssim 1.5$ ps. The measurements and theoretical simulations show important temperature dependences of thermal conductivity, specific heat, and electron-phonon coupling for the highly excited electron-lattice system. For $\mathrm{Au}$, the $5 d$ electrons are thermalized very rapidly with the conduction electrons. The technique presented here is widely applicable to other materials and possibly to higher- $T_{e}$ regimes. An extension of experiments on thin $\mathrm{Au}$ films would further resolve the roles of $\kappa$ and $G$ in the electron cooling dynamics.

We are grateful to J. L. Erskine for providing the UHV chamber used in these experiments. This work was supported by the Air Force Office of Scientific Research (Contract No. F49620-92-C-0027), the National Science Foundation (Grant No. DMR-8858388), and the Robert A. Welch Foundation (Grant No. F-1038).
*Present address: Columbia Radiation Laboratory, Columbia University, New York, NY 10027.

tPresent address: Department of Physics, Utah State University, Logan, UT 84322.

${ }^{1}$ G. L. Eesley, Phys. Rev. Lett. 51, 2140 (1983); S. D. Brorson, J. G. Fujimoto, and E. P. Ippen, ibid. 59, 1962 (1987); R. W. Schoenlein et al., ibid. 58, 1680 (1987); H. E. Elsayed-Ali et al., ibid. 58, 1212 (1987); P. B. Corkum et al., ibid. 61, 2886 (1988); S. D. Brorson et al., ibid. 64, 2172 (1990); H. E. Elsayed-Ali et al., Phys. Rev. B 43, 4488 (1991); T. Juhasz et al., ibid. 45, 13819 (1992).

${ }^{2}$ R. H. M. Groeneveld, R. Sprik, and A. Lagendijk, Phys. Rev. Lett. 64, 784 (1990); Phys. Rev. B 45, 5079 (1992).

${ }^{3}$ J. G. Fujimoto et al., Phys. Rev. Lett. 53, 1837 (1984).

${ }^{4}$ S. M. Sze, Physics of Semiconductor Devices (Wiley, New York, 1969).

${ }^{5}$ H. Shichijo and K. Hess, Phys. Rev. B 23, 4197 (1981); J. M.

Higman et al., J. Appl. Phys. 65, 1384 (1989).

${ }^{6}$ M. M. Murnane et al., Science 251, 531 (1991).

${ }^{7}$ R. M. More et al., Phys. Fluids 31, 3059 (1988); F. Perrot and M. W. C. Dharma-wardana, Phys. Rev. A 36, 238 (1987).

${ }^{8}$ H. M. Milchberg et al., Phys. Rev. Lett. 61, 2364 (1988).

${ }^{9}$ S. Kato, R. Kawakami, and K. Mima, Phys. Rev. A 43, 5560 (1991); M. W. C. Dharma-wardana and F. Perrot, Phys. Lett. A 163, 223 (1992).

${ }^{10}$ F. Budde et al., Phys. Rev. Lett. 66, 3024 (1991).

${ }^{11}$ D. M. Riffe, X. Y. Wang, M. C. Downer, D. L. Fisher, T. Tajima, J. L. Erskine, and R. M. More, J. Opt. Soc. Am. B 10, 1424 (1993).

${ }^{12}$ W. M. Wood, G. Focht, and M. C. Downer, Opt. Lett. 13, 984
(1988).

${ }^{13}$ Optical Properties of Metals, edited by J. H. Weaver et al., (Karlsruhe, Germany, 1981).

${ }^{14} \mathrm{X}$. Y. Wang et al., in Short Wavelength V: Physics with Intense Laser Pulses, edited by M. D. Perry and P. B. Corkum (Optical Society of America, Washington, D.C., 1993).

${ }^{15}$ Since the relationship between $T_{\text {peak }}$ and the laser-pulse intensity is not strictly linear, the shape of $T_{e}(t)$ (from one laser pluse) and $T_{\text {peak }}(\Delta t)$ will not be exactly proportional, and must be obtained by solving the nonlinear coupled equations discussed below [Eqs. (2) and (3)].

${ }^{16}$ W. S. Fann, R. Storz, H. W. K. Tom, and J. Bokor, Phys. Rev. B 46, 13592 (1992); M. B. Agranat, S. I. Anisimov, and B. I. Makshantsev, Appl. Phys. B 47, 209 (1988).

${ }^{17}$ American Institute of Physics Handbook, 3rd ed., edited by D. E. Gray (McGraw-Hill, New York, 1973).

${ }^{18}$ G. K. Wertheim and D. N. E. Buchanan, Phys. Rev. B 33, 914 (1986).

${ }^{19}$ P. B. Allen, Phys. Rev. Lett. 59, 1460 (1987).

${ }^{20}$ N. W. Ashcroft and N. D. Mermin, Solid State Physics (Holt, Rinehart and Winston, New York, 1976), p. 52.

${ }^{21}$ At $k_{B} T_{e} \lesssim 1 \mathrm{eV}$, the change of $\mu$ is only a few percent from $\varepsilon_{F} \sim 5.5 \mathrm{eV}$.

${ }^{22} \mathrm{R}$. Berman, Thermal Conduction in Solids (Clarendon, Oxford, 1976).

${ }^{23}$ A. H. MacDonald, Phys. Rev. Lett. 44, 489 (1980); M. Kaveh and N. Wiser, Adv. Phys. 33, 259 (1984).

${ }^{24}$ S. D. Brorson, J. G. Fujimoto, and E. P. Ippen, Phys. Rev. Lett. 59, 1962 (1987). 